\section{ORIGINAL RESEARCH}

\author{
J. Fruehwald-Pallamar \\ P. Szomolanyi \\ N. Fakhrai \\ A. Lunzer \\ M. Weber \\ M.M. Thurnher \\ M. Pallamar \\ S. Trattnig \\ D. Prayer
}

I.M. Noebauer-Huhmann

\title{
Parallel Imaging of the Cervical Spine at 3T: Optimized Trade-Off between Speed and Image Quality
}

BACKGROUND AND PURPOSE: Patients with cervical spine syndrome often experience pain during the MR examination. Our aim was to compare the quality of cervical spine MR images obtained by parallel imaging with those of nonaccelerated images, with the goal of shortening the examination time while preserving adequate image quality.

\begin{abstract}
MATERIALS AND METHODS: A phantom study and examinations of 10 volunteers and 26 patients were conducted on a clinical 3T scanner. Acquisitions included axial T2WI, sagittal T2WI, T1WI, and T2TIRM sequences. Nonaccelerated sequences and accelerated sequences with different numbers of averages and different accelerations, with a scanning time reduction of $67 \%$, were performed. For quantitative analysis, the SNR was obtained from the phantom measurements, and the NU was calculated from the volunteer measurements. For qualitative analysis, 3 independent readers assessed the delineation of anatomic structures in volunteers and the visibility of degenerative disease in patients.
\end{abstract}

RESULTS: In the phantom study, as expected, the SNR of the nonaccelerated images was higher than the SNR of the same sequence with parallel imaging. In vivo, the NU was higher when applying fewer averages or parallel imaging, compared with the nonaccelerated images. The analysis of the subjective parameters in the volunteers and patients showed that a scanning time of $48 \%$ of the original protocol could be obtained by combining the following sequences: sagittal T1WI with 1 average; sagittal T2WI with acceleration factor 3; sagittal T2TIRM with acceleration factor 2; and axial T2* GRE with acceleration factor 2

CONCLUSIONS: Parallel imaging of the cervical spine at 3T allows shortening of the examination time by $52 \%$, preserving adequate image quality.

ABBREVIATIONS: CNR = contrast-to-noise ratio; GRAPPA = generalized autocalibrating partially parallel acquisition; $\mathrm{GRE}=$ gradient recalled-echo; $\mathrm{NU}=$ nonuniformity; $\mathrm{pMRI}=$ parallel $\mathrm{MR}$ imaging; T2TIRM $=$ T2 turbo inversion-recovery magnitude

U to $50 \%$ of individuals older than 50 years of age have some degree of disk degeneration. ${ }^{1,2}$ Patients are symptomatic due to radiculopathy, cervical myelopathy, or muscular and ligamentous imbalance, as well as such sequelae as osteochondrosis and facet joint osteoarthritis. The annual incidence of cervical radiculopathy is reportedly 83 per 100,000 population, whereas the prevalence is 3.5 per 1000 population, with a peak incidence in the sixth decade of life. ${ }^{3-5}$

In patients with symptoms resistant to therapy or inconclusive findings on projection radiographs, MR imaging is the technique of choice for the evaluation of the cervical spine. The cervical spine bodies, joints, disks, and ligaments, as well as the spinal cord, the nerve roots, and the surrounding soft-

Received November 14, 2011; accepted after revision February 1, 2012.

From the Subdivision of Neuroradiology and Musculoskeletal Radiology (J.F.-P., N.F., M.M.T., D.P., I.M.N.-H.), Department of Radiology; University Clinic of Orthopaedic Surgery (A.L.); Department of Radiology (M.W.); Center of High-Field MRI (P.S., M.P., S.T., I.M.N.-H.,), Department of Radiology; Medical University of Vienna, Vienna, Austria; and Department of Imaging Methods (P.S.), Institute of Measurement Science, Slovak Academy of Sciences, Bratislava, Slovakia.

Please address correspondence to Julia Fruehwald-Pallamar, MD, Department of Radiology, Subdivision of Neuroradiology and Musculoskeletal Radiology, Medical University of Vienna, Waehringer Guertel 18-20, 1090 Vienna, Austria; e-mail: Julia.fruehwald-pallamar@ meduniwien.ac.at

Indicates article with supplemental on-line tables.

Indicates article with supplemental on-line figure.

http://dx.doi.org/10.3174/ajnr.A3101 tissue, can be assessed with MR imaging. For sufficient image quality, patients must avoid any movement that might cause motion artifacts. For patients with painful spine conditions, in particular, it is important to reduce the examination time while still obtaining diagnostic images. One possibility for decreasing scanning time is to reduce the number of acquisitions. Another technique, pMRI, became possible with the introduction of multicoil arrays. ${ }^{6}$ In the past few years, pMRI has been increasingly used to accelerate MR images and reduce scanning time. ${ }^{7-13}$ In pMRI, compared with conventional MR imaging, the number of phase-encoding steps in the same FOV can be reduced, with an array of multiple independent receiver coils that acquire signals simultaneously.

With the GRAPPA technique, it is possible to omit phaseencoding steps from the abundant information received from the various coil elements during acquisition. ${ }^{14}$

Several studies, mostly on $1.5 \mathrm{~T}$ scanners, have proved that with the use of parallel imaging, the examination time can be reduced substantially while preserving good image quality. ${ }^{15-17}$ The benefits of a shorter breath-hold time for pMRI has been proved in cardiac, thoracic, and liver imaging ${ }^{16,18}$ and even with free breathing in cardiac MR imaging. ${ }^{19}$ In contrast-enhanced MR angiography, pMRI is used to achieve a higher temporal resolution. In general, the higher SNR of 3T systems can be used for better image quality or reduced scanning time. It is possible to test higher acceleration factors on 


\begin{tabular}{|c|c|c|c|c|}
\hline \multicolumn{5}{|c|}{ Imaging parameters and examination times } \\
\hline Sequence & Sagittal T1WI & Sagittal T2WI & Sagittal T2TIRM & Axial T2* GRE \\
\hline TR (ms) & 600 & 3000 & 3000 & 1300 \\
\hline TE (ms) & 10 & 109 & 32 & 14 \\
\hline Flip angle & $160^{\circ}$ & $180^{\circ}$ & $140^{\circ}$ & $30^{\circ}$ \\
\hline Section thickness (mm) & 3 & 3 & 3 & 3 \\
\hline Gap (mm) & 3.3 & 3.3 & 3.3 & 3.3 \\
\hline NEX & 3 & 2 & 1 & 1 \\
\hline ETL & 53 & 31 & 7 & 1 \\
\hline Matrix (reconstruction) & $384 \times 306$ & $320 \times 320$ & $640 \times 640$ & $512 \times 512$ \\
\hline Matrix (acquisition) & $384 \times 260$ & $320 \times 256$ & $320 \times 240$ & $256 \times 256$ \\
\hline FOV & $191 \times 240$ & $240 \times 240$ & $240 \times 240$ & $160 \times 160$ \\
\hline \multicolumn{5}{|l|}{ Time (min/sec) } \\
\hline Nonaccelerated (original) & 03:10 & 03:06 & 03:24 & 08:19 \\
\hline 2 Averages (\% reduction) & $02: 06(33.7 \%)$ & & & \\
\hline 1 average & $01: 03(67 \%)$ & $01: 30(54 \%)$ & & \\
\hline Acc 2 & $01: 36(49.5 \%)$ & $01: 31(53.4 \%)$ & $01: 48(47 \%)$ & $04: 24(47 \%)$ \\
\hline Acc 3 & $01: 04(66.4 \%)$ & $01: 07(66 \%)$ & $01: 15(63 \%)$ & 03:06 (63\%) \\
\hline Acc 4 & $01: 00(68.8 \%)$ & $00: 55(67.6 \%)$ & $00: 57(72 \%)$ & $02: 27(74.5 \%)$ \\
\hline
\end{tabular}

Note:-ETL indicates echo-train length; Acc, acceleration factor.

higher than $1.5 \mathrm{~T}$ units. Imaging of the cervical spine should benefit from the use of higher field strengths in terms of scanning time reduction, especially when dealing with patients who cannot lie still for a long time.

However, to apply this technique routinely in cervical spine imaging, we had to evaluate the image quality and clinical utility at $3 \mathrm{~T}$ systematically. The aim of our study was, therefore, to apply parallel imaging at $3 \mathrm{~T}$ in cervical spine imaging. For this purpose, we tested accelerated and nonaccelerated sequences objectively and subjectively in a phantom, in volunteers, and in patients.

\section{Materials and Methods}

The MR imaging examination was performed on a $3 \mathrm{~T}$ clinical scanner (Tim Trio; Siemens, Erlangen, Germany) with a maximum gradient strength of $38 \mathrm{mT} / \mathrm{m}$ and a gradient slew rate of $170 \mathrm{mT} / \mathrm{m} / \mathrm{ms}$. We used an array of dedicated head, spine, and neck coils. For sagittal measurements (phantom, volunteers, and patients), the standard neck coil (3T Neck Matrix, a total imaging matrix coil; Siemens) and the standard head coil (3T Head Matrix, a total imaging matrix coil; Siemens) were used. All axial measurements were performed with the standard spine coil (3T Spine Matrix, a total imaging matrix coil; Siemens).

First, a phantom study was conducted to evaluate the protocol and to assess the SNR of the accelerated and nonaccelerated sequences. Acquisitions included axial T2*-weighted sequences $(2 \mathrm{D}$ spoiled gradient-echo multiecho sequences ${ }^{20}$ ), sagittal T2-TSE, sagittal T1-TSE, and sagittal T2TIRM sequences. Detailed sequence parameters are listed in the Table.

Those 4 sequences were measured by using GRAPPA, with an acceleration factor of 1-4, as well a reduced number of averages (sagittal T2WI, sagittal T1WI).

We examined 1 cylindric phantom (a plastic bottle filled with 3.7-g nickel sulphate $\times 6 \mathrm{H}_{2} \mathrm{O}+5$-g sodium chloride per 1000- $\mathrm{g} \mathrm{H}_{2} \mathrm{O}$, distilled; the signal intensity of the content mimics CSF), 10 healthy volunteers (mean age, 35 years; range, 19-53 years), and 26 patients with pain and/or neurologic deficits due to suspected degeneration (mean age, 46 years; range, $21-75$ years). In the phantom study, the image quality of the sequence with an acceleration factor of 4 was insufficient (low SNR). Consequently, all volunteers and patients were only examined with acceleration factors of 2 and 3.

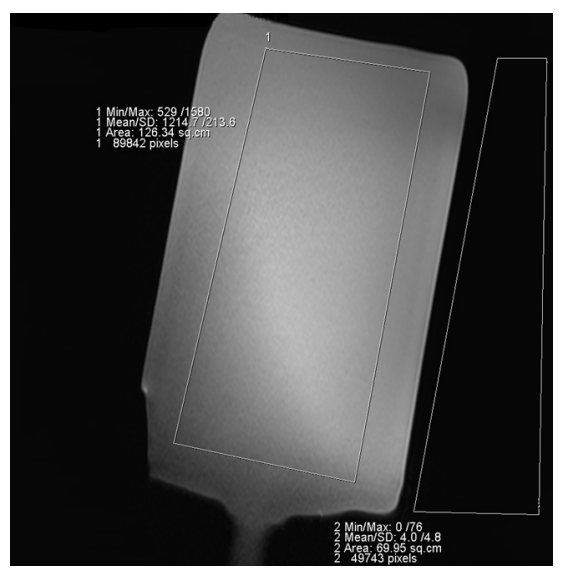

Fig 1. Region-of-interest measurements in the phantom.

The institutional ethics committee approved this study, and written, informed consent was obtained from the 10 volunteers and 26 patients.

\section{Quantitative: Phantom}

For quantitative analysis, the SNR was calculated for each sequence and acceleration factor from the phantom measurements. A large region of interest covering major parts of the phantom (corresponding to the location of relevant structures in vivo) and a second region of interest placed on the background next to the phantom were drawn on an image in the middle of the stack (Fig 1). The ROIs were drawn large to average the $B_{1}$ inhomogeneity in the center of the phantom.

SNR was calculated by a modified dual acquisition $\operatorname{method}^{8}$ by using the following formula:

$$
S N R=\sqrt{2 \frac{S I}{S D_{S I 1-S I 2}}},
$$

where $S I$ is the signal intensity of a region of interest placed on an image of the phantom in the middle of the stack, and $S D_{S I 1-S I 2}$ is the $\mathrm{SD}$ of the signal intensity of the same region of interest on the "subtracted image," representing the noise. 


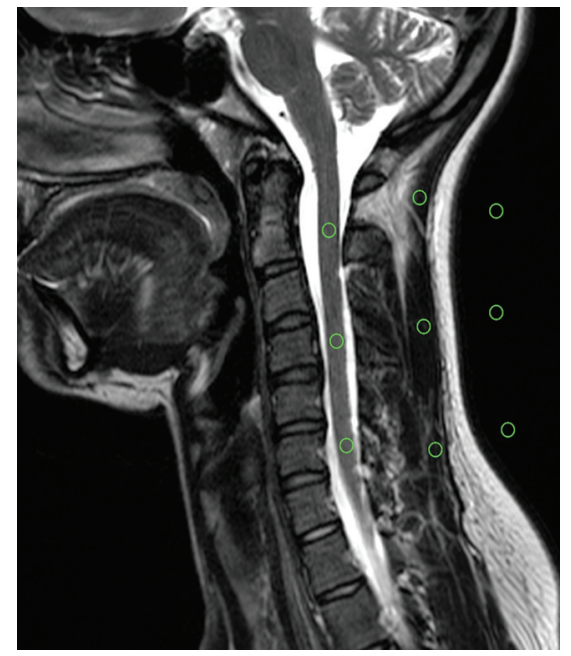

Fig 2. The region-of-interest measurements in a volunteer.

\section{Quantitative: Volunteers}

In the volunteer examinations, 3 ROIs (levels C2, C4, and C6) were placed in the spinal cord $(s c)$, in the paravertebral muscle $(m)$, and in the background air in a central section of the stack (Fig 2). From these measurements, the NU was calculated by using the following formula:

$$
N U=\frac{S D_{s c}}{S I_{s c}} \times 100,
$$

with $S I_{\mathrm{sc}}$ taken as the mean signal intensity of the ROIs in the spinal cord, and $S D_{s c}$ taken as the $S D$ of these ROIs.

In the 10 volunteers, the CNR was also calculated by

$$
C N R=\frac{S I_{s c}-S I_{m}}{S D_{a i r}},
$$

where $S I_{s c}$ is the mean signal intensity measured by the ROIs in the spinal cord, $S I_{m}$ is the mean signal intensity of the ROIs measured in the muscles, and $S D_{\text {air }}$ is the $S D$ of the ROIs in the air.

\section{Qualitative: Volunteers}

Qualitative analysis was performed by 3 independent readers (J.F.-P., I.M.N.-H., N.F., who had MR imaging experience of 5, 14, and 4 years, respectively), on a commercial PACS workstation. The readers were blinded to the technique and volunteer/patient name. They interpreted the images in a random order.

In the volunteer examinations, the delineation of 6 anatomic structures (facet joints, central spinal canal, neural foramina, nerve roots, disks, spinal cord) was rated as 5 (excellent visibility), 4 (good), 3 (acceptable), 2 (poor), or 1 (not visible). The overall subjective impression of the image was assessed in the same manner. Artifacts (motion, aliasing, susceptibility, section overlap) were graded as absent (5), minimal (4), mild (3), moderate (2), or severe (1). We determined that a sequence with $>10 \%$ of "problematic" ratings ( 1 or 2 ) should be excluded from the protocol. For this calculation, we used the total number of ratings (all readers and patients/volunteers).

\section{Qualitative: Patients}

In the patients, we evaluated the following degenerative changes: abnormal posture, disk degeneration, disk herniations, Modic changes, spondylophytes, facet arthropathy, spinal stenosis, neural foramina stenosis, and compression myelopathy. "Disk degeneration" was defined as height and signal loss on the sagittal T2 and turbo-inversion
Non Uniformity

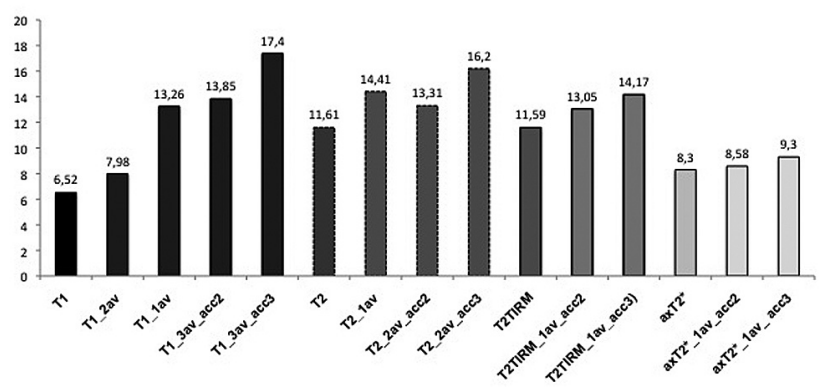

Fig 3. The mean nonuniformity of the 10 volunteers for each sequence.

recovery magnitude sequences. "Disk herniation" was defined as focal extension of the disk past the vertebral body. Endplate changes were defined according to the classification of Modic and Ross. ${ }^{21}$ Facet joint degeneration and neural foramina stenosis were evaluated according to the criteria described by Morvan. ${ }^{22}$ There is no consensus definition of cervical spinal canal stenosis. Cervical spinal canal stenosis was defined as the absence of CSF signal around the spinal cord. Myelopathy was defined as high signal changes in the spinal cord on the T2-weighted sequences.

The 3 readers rated the visibility of pathology as 5 (excellent visibility), 4 (good), 3 (acceptable), 2 (poor), or 1 (not visible). A cutoff at $10 \%$ of missed pathologies was defined for exclusion of an accelerated sequence.

Data were analyzed by using the Statistical Package for the Social Sciences for Windows (Version 17.0; SPSS, Chicago, Illinois). Nominal data are presented by using absolute frequencies and percentages. Metric data are presented by using mean \pm SD when normal and distributed and median (minimum, maximum) when skewed. To compare the original with the accelerated sequences with respect to SNR, CNR, and NU, we used 1-way ANOVAs for repeated measures, followed by simple contrasts in case of significant results. A $P$ value $\leq$ .05 indicated a significant result. As corrections for multiple comparisons decrease the power to detect a difference, no such corrections were applied.

\section{Results}

\section{Quantitative: Phantom}

In the phantom study, the SNR of the nonaccelerated images was higher than the SNR of the same sequence with parallel imaging (On-Line Table 1).

\section{Quantitative: Volunteers}

In vivo, the NU was higher with parallel imaging with an acceleration factor of 2 or 3 ; or 1 average in the sagittal T1WI and an acceleration factor of 3 ; or 1 average in the sagittal T2WI and an acceleration factor of 3 in the T2TIRM sequence, compared with the nonaccelerated images (Fig 3 ). For the sagittal T1WI (3 averages) sequence, we found significant differences in the NU between the original sequence and the T1WI (1 average), T1WI acceleration factor 2, and T1WI acceleration factor $3(P>.05)$. The T2TIRM acceleration factor 3 had a significantly higher NU than the nonaccelerated sequence. All 

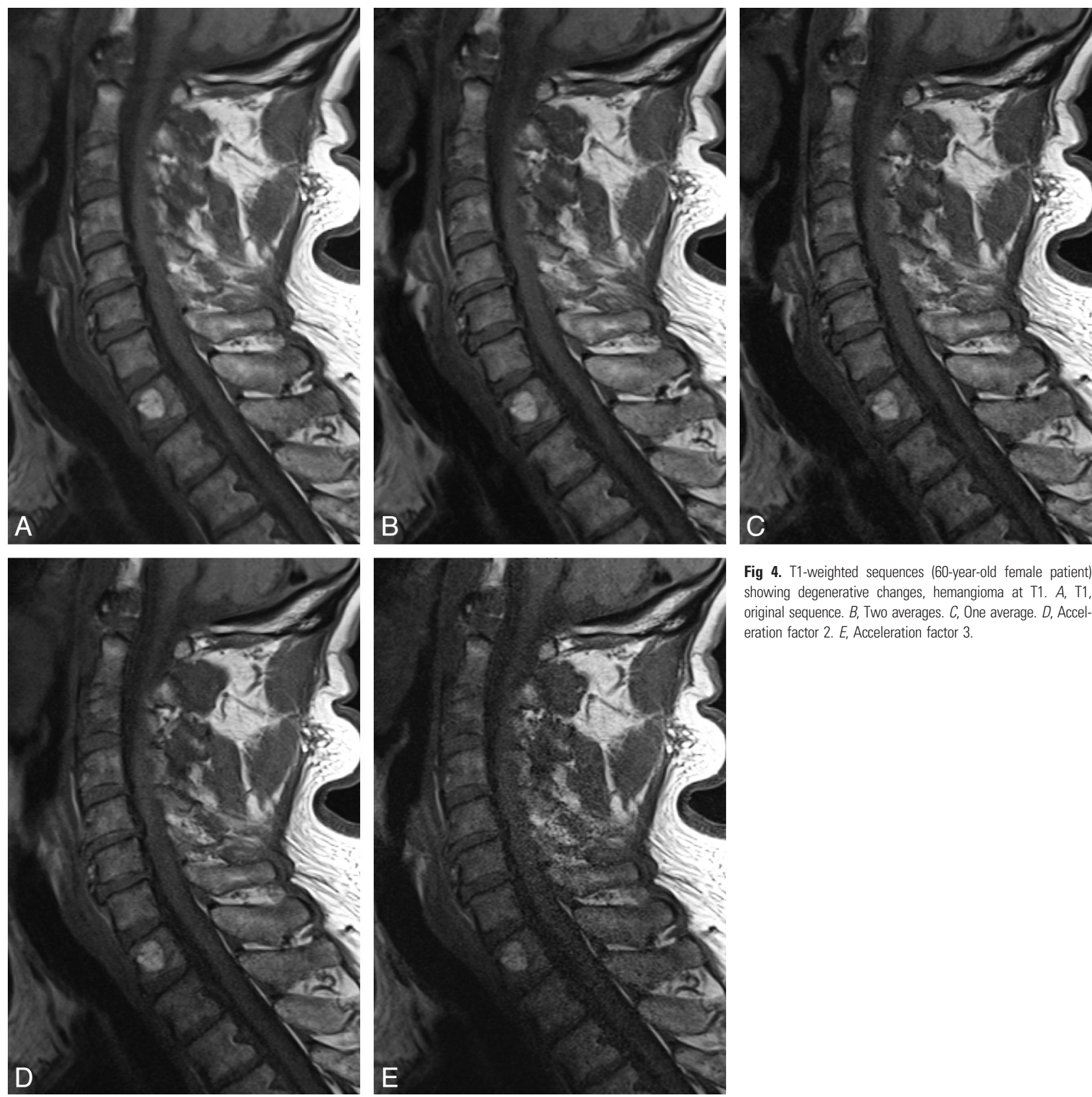

Fig 4. T1-weighted sequences (60-year-old female patient) showing degenerative changes, hemangioma at $\mathrm{T} 1 . A, \mathrm{~T} 1$, original sequence. $B$, Two averages. $C$, One average. D, Acceleration factor 2. E, Acceleration factor 3.

$\mathrm{NU}$ values and differences between the original and accelerated sequences can be found in On-Line Table 2.

With regard to the CNR, as expected, we did not find significant differences in any accelerated sequence compared with the original sequence, with the exception of the sagittal $\mathrm{T} 2$ sequence group, in which the original and the T2WI acceleration factor 3 differed $(P=.003)$.

\section{Qualitative: Volunteers}

Because of severe motion artifacts, we excluded volunteer 8 from the statistics. In the other 9 volunteers, all 6 important anatomic structures were at least adequately visible (grade III) in all sequences, except for the nerve roots and the spinal cord in the 3-fold acceleration factor T1WI and the 3-fold acceleration factor T2TIRM images. By calculating the percentages of the ratings 1 and 2, we analyzed all subjective parameters (6 anatomic structures, artifacts, and overall image quality).
Only in the T1, T2, and T2TIRM with an acceleration factor of 3 did we find ratings of 1 (in total 8 times for all readers in all volunteers). The percentages of problematic ratings 1 and 2 are listed in On-Line Table 1. We also determined that a sequence with $>10 \%$ of problematic ratings had to be excluded from the protocol. We had to exclude the sagittal T1WI sequence with acceleration factor 3 and the sagittal T2TIRM sequence with acceleration factor 3 .

\section{Qualitative: Patients}

In 26 patients, we found the following pathologies: 24 patients with at least 1 desiccated disk, 1 with a hyperintensity zone; and 16 disk herniations, 7 of which caused spinal canal stenosis and 5 of which caused compression of the spinal cord. One patient of the latter group showed signs of compression myelopathy. Twenty patients had spondylophytes, and 7 had Modic type 1 endplate changes. Twelve patients had at least a 

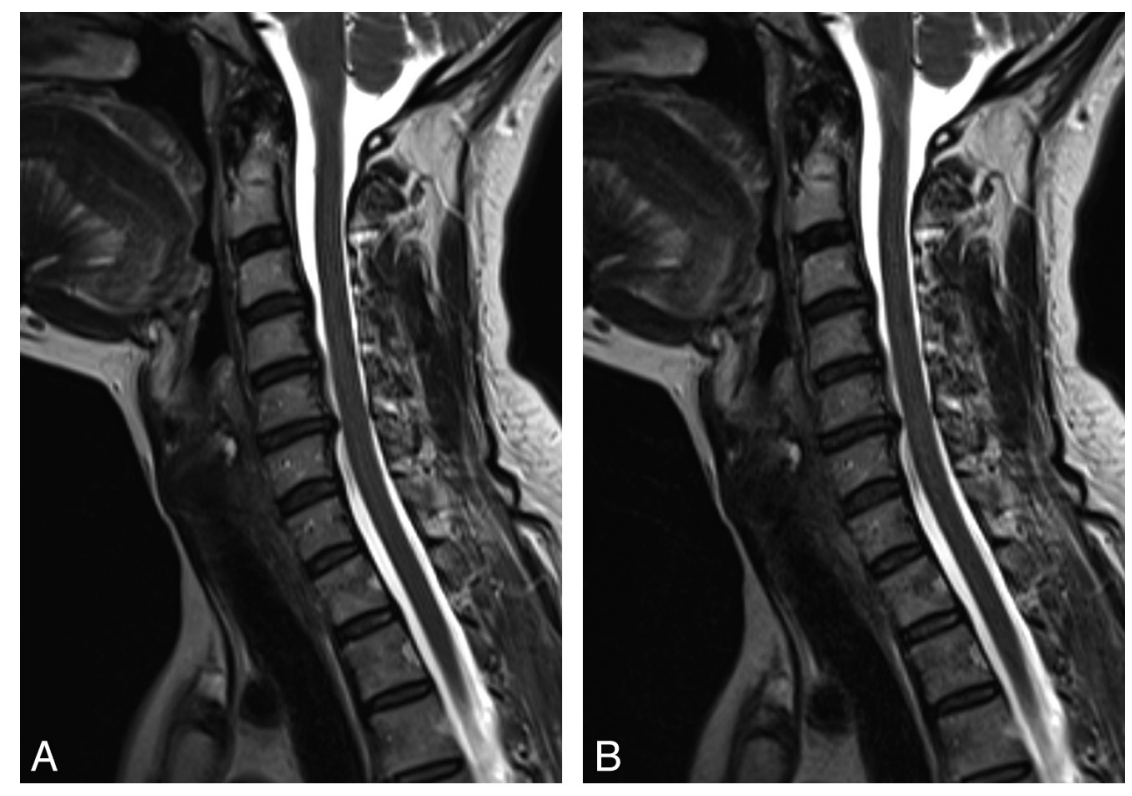

Fig 5. T2-weighted sequences (53-year-old female volunteer) showing a small hernia of the $\mathrm{C} 5 / 6$ disk. $A$, T2, original sequence. $B$, One average. C, Acceleration factor 2. $D$, Acceleration factor 3 .
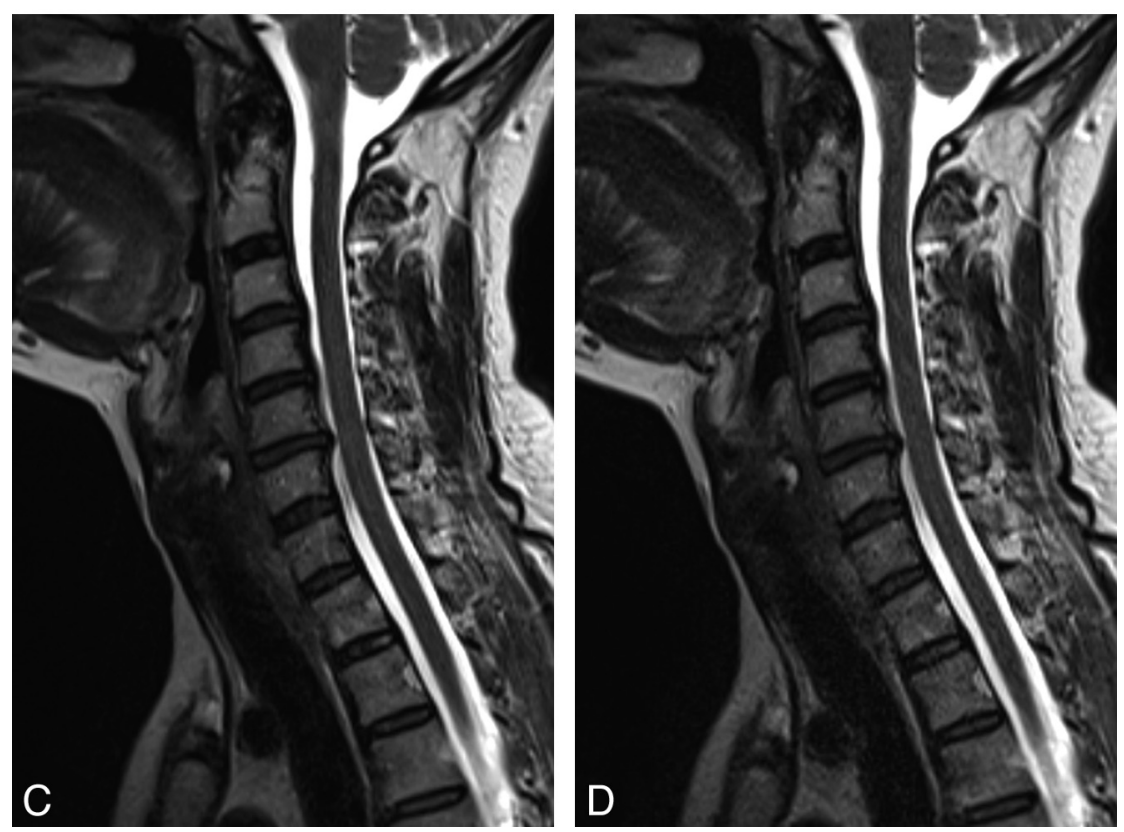

neuroforaminal stenosis at 1 level. One patient showed an intravertebral hernia; 1, a hemangioma (Fig 4); and 1, a subcutaneous lipoma. In 20/26 patients, we found abnormal posture.

For the patient analyses, we calculated how often a finding was missed in the accelerated sequence compared with the original sequence. We also defined a cutoff at $10 \%$ of missed pathologies for a sequence to be excluded. The results are listed in On-Line Table 2. We consequently excluded the sagittal T1WI sequence with acceleration factor 3 and the sagittal T2TIRM sequence with acceleration factor 3. The T2TIRM sequence with acceleration factor 2 was $10.2 \%$ in the volunteer evaluation and $6.8 \%$ in the patient evaluation.

When comparing qualitative parameters (NU) with our subjective evaluation, the highest NU (On-Line Table 2) was seen in the sequences with the highest acceleration factor. These sequences also had more problematic ratings (On-Line Table 3) or missed pathologies (On-Line Table 4).

\section{Discussion}

In recent years, great progress in pMRI methods has allowed the use of these techniques in a variety of MR imaging applications. ${ }^{23}$ The present study evaluates the effect of a parallel imaging technique for the cervical spine and the modification of scanning time by reducing the averages. We showed that the scanning time can be reduced up to $50 \%$ while preserving adequate image quality. Poorer image quality at higher acceleration factors affected diagnostic performance.

Parallel imaging can be applied to shorten the acquisition time, especially in breath-hold sequences; to lessen the total examination time; or to increase either the SNR or spatial resolution at a constant imaging time.

At $1.5 \mathrm{~T}$, several studies demonstrated the potential of parallel imaging to reduce the scanning time in vertebral spine imaging. ${ }^{15,17}$ With the introduction of $3 \mathrm{~T}$ high-field MR imaging units, along with increased SNR, a further reduction of scanning time with the use of parallel imaging can be expected. 

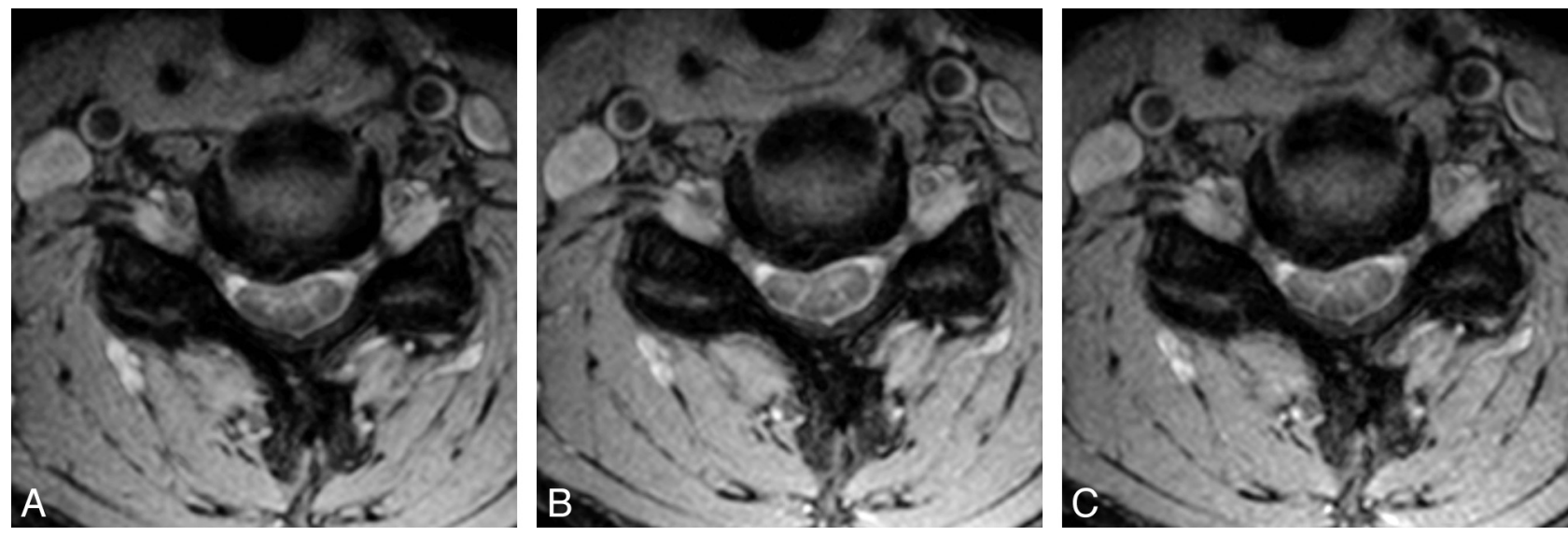

Fig 6. Axial T2* GRE sequences (46-year-old female patient) showing posterolateral disk herniation with compression of the spinal cord. $A$, T2* GRE, original sequence. $B$, Acceleration factor 2 . C. Acceleration factor 3

Further advantages are the stronger gradients and new coil technologies, which also increase SNR, with the potential of testing higher acceleration factors than is possible on $1.5 \mathrm{~T}$. Noebauer-Huhmann et al ${ }^{15}$ as well as Nölte et $\mathrm{al}^{17}$ were able to apply an acceleration factor of 2 for their cervical/lumbar spine protocol at $1.5 \mathrm{~T} .{ }^{15,17} \mathrm{With}$ the use of $3 \mathrm{~T}$, we were able to test even higher acceleration factors up to 4 . Our study is a translational study that includes phantom measurements, volunteers, and patients. Noebauer-Huhmann et al measured a phantom and 10 volunteers, and Nölte et al examined 31 patients. The disadvantages of higher field strengths are a higher inhomogeneity and images that are more prone to artifacts. Particularly in patients who have undergone cervical fusion, susceptibility artifacts can significantly impair image quality. Furthermore, some specific metals are contraindicated for use at $3 \mathrm{~T}$.

To date, several studies concerning technical factors and in vitro image quality have been published, with only a few studies examining the feasibility in patients. To our knowledge, no such study has been performed in cervical spine imaging at $3 \mathrm{~T}$.

In the phantom measurements, as expected and known from the literature, ${ }^{24}$ the SNR of the accelerated sequences was lower than that in the original sequence. Other authors have also reported a decrease of up to $35 \%$ SNR when applying parallel imaging. ${ }^{16}$ Because of the inhomogeneous distribution of noise over the FOV after the integrated parallel acquisition technique reconstruction, we did not perform SNR measurements in the in vivo examinations in volunteers or patients ${ }^{25,26}$ but used the nonuniformity to assess the image quality in the volunteers, as recommended in the literature. ${ }^{15,27}$ Inhomogeneous signal and motion artifacts result in a high NU. To reduce the effect of inhomogeneous noise over the FOV, we measured ROIs from different levels (C2, C4, and C6). ${ }^{15}$ We could see increased NU in the accelerated sequences, compared with the original sequences. Reducing the averages had a lower impact on the NU than applying acceleration factors. In the axial $\mathrm{T}^{\star}$ GRE sequence, the increase in $\mathrm{NU}$ was the lowest among the 4 sequences.

In the subjective evaluation of the volunteers, we excluded sequences with $>10 \%$ ratings of 1 or 2 (not visible, poor) for the visibility of the defined anatomic structures. The acceleration factor 3 T1WI and T2TIRM could be clearly excluded. In the T2TIRM acceleration factor 2, we found $10.2 \%$ of ratings 1 and 2, but we still included this sequence because of the good objective parameters, the good performance in the patient evaluation (only $6.8 \%$ missed pathologies), and the fact that in the original sequence, 8 problematic rates were also found. Thus, in comparison with the original sequence, the T2TIRM acceleration factor 2 showed insufficient diagnostic quality in an additional $6.5 \%$ of cases. The other nonaccelerated sequences showed insufficient diagnostic quality in $<1 \%$. When we evaluated pathologic findings, we also clearly could exclude the T1WI acceleration factor 3 and the axial T2 ${ }^{\star}$ GRE acceleration factor 3 . In the patient examinations, 1 sequence was marginally at the cutoff-the axial T2* GRE acceleration factor 2 with $10.2 \%$ rated as grade 1 or 2 . This sequence had a very good SNR and low NU and only 1 problematic rate in the volunteers. Consequently, we decided to include this sequence in the protocol.

If we took those results into account, the fastest sequences of the new protocol with sufficient image quality included the following: sagittal T1WI 1 average (1 minute, 3 seconds); sagittal T2WI sequence with acceleration factor 3 (1 minute, 7 seconds); sagittal T2TIRM sequence with acceleration factor 2 (1 minute, 48 seconds); and the axial T2* GRE sequence with acceleration factor 2 ( 4 minutes, 24 seconds). This protocol takes 8 minutes 22 seconds, $48 \%$ of the original protocol, which had a duration of 17 minutes 59 seconds. Examples are given in Figs 4-7.

In daily clinical routine, pMRI is used to reduce scanning time in sequences that require breath-holding. We see the potential application for this shorter time protocol of the spine in noncompliant patients and in patients who find it painful to lie in the magnet (eg, trauma patients). With parallel imaging, it can be assumed that shorter sequences are less prone to artifacts and that motion artifacts decrease, which is another advantage when imaging patients who find it difficult to lie still. $^{28}$

A limitation of our study was the lack of a histologic correlation of the pathologic findings, which was not possible in this study group. A further limitation is the small number of 26 patients, but we think we have proved the clinical feasibility of parallel imaging by analyzing additional anatomic details in 

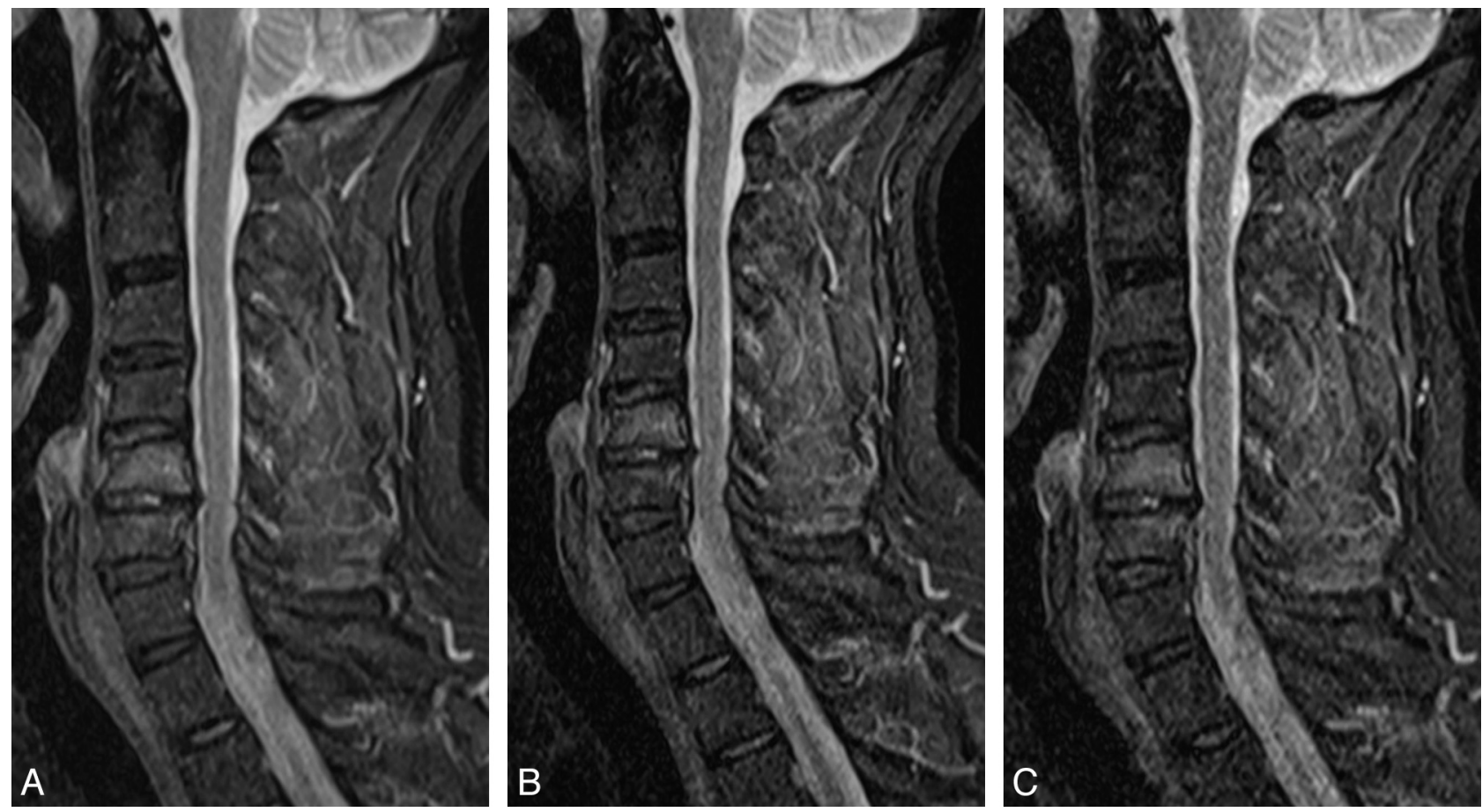

Fig 7. T2TIRM sequences (45-year-old male patient) showing slight edema of C5 due to disk degeneration and minor endplate edema of C5 (Modic type I). A, T2TIRM, original sequence. B, Acceleration factor 2. C, Acceleration factor 3.

the volunteers, which increased the study population to 36 individuals.

Several manufacturers have developed parallel imaging techniques. We used a single MR imaging unit for our study; therefore, the results cannot be transferred/compared with other parallel imaging techniques. In addition, the specific settings of coils, receiver channels, and scanner-specific parameters provided by the manufacturer must be considered when comparing our results with those in other units/systems.

\section{Conclusions}

In imaging of the cervical spine at 3T, the scanning time (with 4 standard sequences: sagittal T1WI with 1 average [ 1 minute, 3 seconds]; sagittal T2WI sequence with acceleration factor 3 [ 1 minute, 7 seconds]; sagittal T2TIRM sequence with acceleration factor 2 [ 1 minute, 48 seconds]; and the axial T2* GRE sequence with acceleration factor 2 [ 4 minutes, 24 seconds]) can be reduced up to $52 \%$ while preserving adequate diagnostic image quality.

Disclosures: Majda Thurnher-UNRELATED: Royalties: Amirsys. Iris-Melanie NobauerHuhmann — UNRELATED: Payment for Development of Educational Presentations: European School of Radiology (ESOR), Erasmus, European Society for Magnetic Resonance in Medicine and Biology (ESMRMB), Comments: occasional invited educational talks not related to the topic, Travel/Accommodations/Meeting Expenses Unrelated to Activities Listed: ESOR, Erasmus, ESMRMB, Comments: reimbursement for invited talks not related to the topic.

\section{References}

1. Boden SD, McCowin PR, Davis DO, et al. Abnormal magnetic-resonance scans of the cervical spine in asymptomatic subjects: a prospective investigation. J Bone Joint Surg Am 1990;72:1178-84

2. Lehto IJ, Tertti MO, Komu ME, et al. Age-related MRI changes at 0.1 T in cervical discs in asymptomatic subjects. Neuroradiology 1994;36:49-53

3. Cote P, Cassidy JD, Carroll LJ, et al. The annual incidence and course of neck pain in the general population: a population-based cohort study. Pain 2004;112:267-73

4. Radhakrishnan K, Litchy WJ, O'Fallon WM, et al. Epidemiology of cervical radiculopathy: a population-based study from Rochester, Minnesota, 1976 through 1990. Brain 1994;117(Pt 2):325-35

5. Salemi G, Savettieri G, Meneghini F, et al. Prevalence of cervical spondylotic radiculopathy: a door-to-door survey in a Sicilian municipality. Acta Neurol Scand 1996;93:184-88

6. Roemer PB, Edelstein WA, Hayes CE, et al. The NMR phased array. Magn Reson Med 1990;16:192-225

7. Glockner JF, Hu HH, Stanley DW, et al. Parallel MR imaging: a user's guide. Radiographics 2005;25:1279-97

8. Heidemann RM, Ozsarlak O, Parizel PM, et al. A brief review of parallel magnetic resonance imaging. Eur Radiol 2003;13:2323-37

9. Kurihara Y, Yakushiji YK, Tani I, et al. Coil sensitivity encoding in MR imaging: advantages and disadvantages in clinical practice. AJR Am J Roentgenol 2002;178:1087-91

10. Larkman DJ, Nunes RG. Parallel magnetic resonance imaging. Phys Med Biol 2007;52:R15-55

11. Pruessmann KP, Weiger M, Scheidegger MB, et al. SENSE: sensitivity encoding for fast MRI. Magn Reson Med 1999;42:952-62

12. Romaneehsen B, Oberholzer K, Muller LP, et al. Rapid musculoskeletal magnetic resonance imaging using integrated parallel acquisition techniques (IPAT): initial experiences. Rofo 2003;175:1193-97

13. Sodickson DK, Griswold MA, Jakob PM. SMASH imaging. Magn Reson Imaging Clin N Am 1999;7:237-54, vii-viii

14. Griswold MA, Jakob PM, Heidemann RM, et al. Generalized autocalibrating partially parallel acquisitions (GRAPPA). Magn Reson Med 2002;47:1202-10

15. Noebauer-Huhmann IM, Glaser C, Dietrich O, et al. MR imaging of the cervical spine: assessment of image quality with parallel imaging compared to nonaccelerated MR measurements. Eur Radiol 2007;17:1147-55

16. Vogt FM, Antoch G, Hunold P, et al. Parallel acquisition techniques for accelerated volumetric interpolated breath-hold examination magnetic resonance imaging of the upper abdomen: assessment of image quality and lesion conspicuity. J Magn Reson Imaging 2005;21:376-82

17. Nölte I, Gerigk L, Brockmann MA, et al. MRI of degenerative lumbar spine disease: comparison of non-accelerated and parallel imaging. Neuroradiology 2008;50:403-09

18. Jakob PM, Griswold MA, Edelman RR, et al. Accelerated cardiac imaging using the SMASH technique. J Cardiovasc Magn Reson 1999;1:153-57

19. Seiberlich N, Ehses P, Duerk J, et al. Improved radial GRAPPA calibration for real-time free-breathing cardiac imaging. Magn Reson Med 2011;65: 492-505 
20. Held P, Dorenbeck U, Seitz J, et al. MRI of the abnormal cervical spinal cord using 2D spoiled gradient echo multiecho sequence (MEDIC) with magnetization transfer saturation pulse: a T2* weighted feasibility study. J Neuroradiol 2003;30:83-90

21. Modic MT, Ross JS. Lumbar degenerative disk disease. Radiology 2007;245: 43-6

22. Morvan G. Imaging of lumbar stenosis [in French]. J Radiol 2002;83(9 pt 2): 1165-75, discussion 1177-79

23. Blaimer M, Breuer F, Mueller M, et al. SMASH, SENSE, PILS, GRAPPA: how to choose the optimal method. Top Magn Reson Imaging 2004;15:223-36

24. Larkman DJ, Nunes RG. Parallel magnetic resonance imaging. Phys Med Biol 2007;52:R15-55
25. Dietrich O, Reeder S, Reiser M, et al. Influence of parallel imaging and other reconstruction techniques on the measurement of signal-to-noise ratios. In: Proceedings of the $13^{\text {th }}$ Scientifc Meeting and Exhibition of the International Society for Magnetic Resonance Imaging in Medicine, Miami, Florida. May 7-13, 2005

26. Ruel L, Brugières $P$, Luciani A, et al. Comparison of in vitro and in vivo MRI of the spine using parallel imaging. AJR Am J Roentgenol 2004;182:749-55

27. Li T, Mirowitz SA. Comparative study of fast MR imaging: quantitative analysis on image quality and efficiency among various time frames and contrast behaviors. Magn Reson Imaging 2002;20:471-78

28. Nelles M, Konig RS, Gieseke J, et al. Dual-source parallel RF transmission for clinical MR imaging of the spine at 3.0 T: intraindividual comparison with conventional single-source transmission. Radiology 2010;257:743-53 